\title{
Evaluation of Nanometer Cutting Tool Edge for Nanofabrication
}

\author{
Yuki Shimizu, \\ Takemi Asai and Wei Gao \\ Tohoku University, \\ Japan
}

\section{Introduction}

Demands for the nanofabrication of the precision surfaces, which have three- dimensional (3D) structures to add specific functions, are continuously increasing. For the nanofabrication on machining tools such as an ultra-precision turning, an ultra-precision diamond cutting tool is generally employed because of its sharp edge and durability. Figure 1(a) shows the illustrations of a chip of the diamond cutting tool. The diamond chip is mounted on the tip of the cutting tool. Figure 1(b) and (c) show a top view from the rakeface side and a side view of the chip, respectively. The chip has a certain nose (corner) radius $R$ at its edge top as shown in Fig. 1(b). The rake angle, radius of the cutting edge, wedge angle and clearance angle are determined as shown in Fig. 1(c).

In the nanofabrication, a quality of the fabricated surface will be dominated by the accuracy of the cutting edge profile. Although the design of the cutting edge profile has some tolerances for its shape, the allowed tolerances for ultra-precision machining are so tight that the highly-accurate measurement technique would be required for assuring the accuracy of the cutting edge profile.

Moreover, a wear of the tool edge should also be taken into consideration for the nanofabrication. Basically, the amount of the wear is very small because the diamond is a hard material. However, a slight wear of the tool edge cannot be avoided even though target materials are the non-steel alloys; namely, the cutting edge profile could change every short periods of the use. The slight change of the cutting edge profile could affect the accuracy of the fabricated surfaces. Therefore, the cutting edge profile should be checked periodically. Generally, the accurate measurement of the cutting edge profile is a time-consuming task. That is why, not only the accuracy but also the easiness of the measurement setting will be required for the nanometer-level evaluation of the cutting edge profile.

Based on such a background described above, in this chapter, the authors describe a method to evaluate the nanometer cutting tool edge by using AFM, including not only a measurement theory but also experimental setup and some results of the tool edge measurement. 


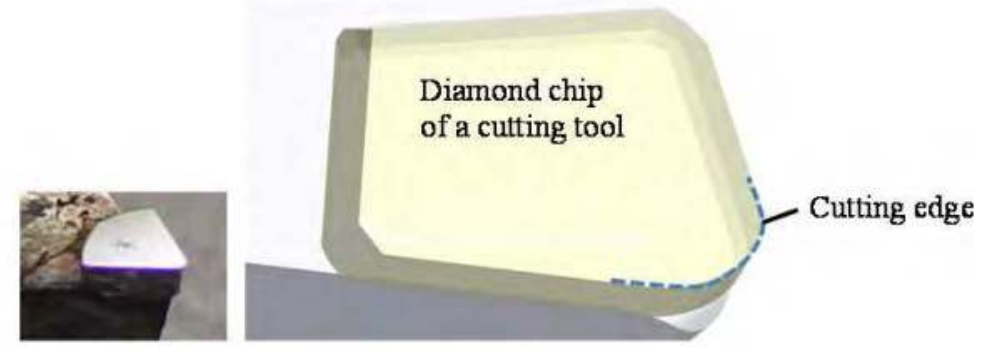

(a) Bird view

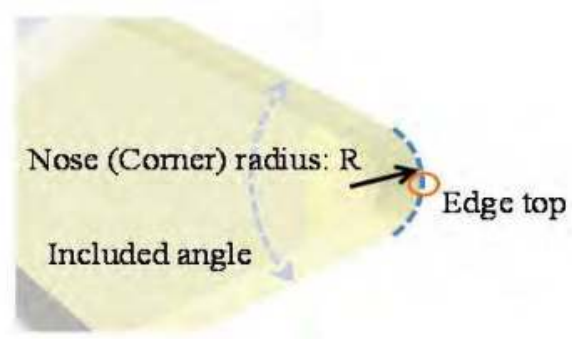

(b) Top view (from the race face side)

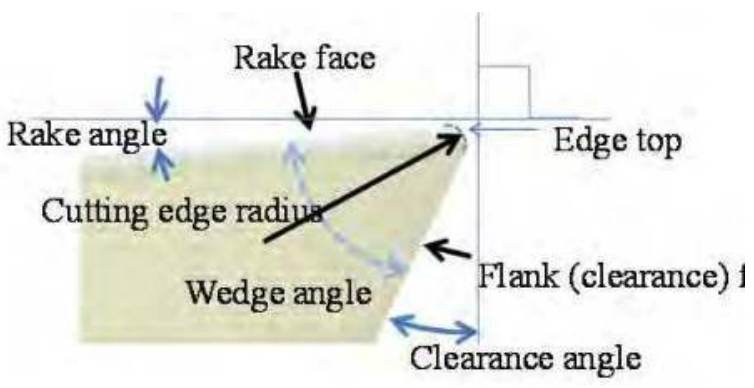

(c) Side view

Fig. 1. Illustrations of a diamond cutting tool chip

\section{A method for compensation of the AFM tip geometry}

\subsection{Cutting edge profile measurement by AFM}

There are several methods to evaluate the cutting edge profile. In most of the cases, scanning electron microscopes (SEMs) are used because of its ease. However, the SEMs require vacuum chambers and vacuum pumps, resulting in bulky systems. On the other hand, the Atomic force microscope (AFM), which will provide a high accuracy and a high resolution in both vertical and lateral directions, could be used for the evaluation of the cutting edge profile until the measurement system would be set properly (Gao et al., 2006). The compact AFM system for the measurement of the cutting edge profile of the diamond tool was previously proposed (Gao, 2010). In the system, because of the simplicity, the contact mode AFM was selected among several measurement mode of the AFM. A unique method was 
also proposed to align the AFM probe with the cutting edge of the ultra-precision diamond cutting tool. Figure 2 shows a schematic of the cutting edge profile measurement by AFM. In the alignment method, a pair of laser beam and photodetector was employed to align tool edge and cantilever of the AFM. By using the compact AFM system with the unique alignment method, the cutting edge profile could successfully be carried out on-machine.

The cutting edge profiles measured by the AFM are affected by the tip shape of the AFM probe. For nanofabrication, the influence of the shape of the AFM probe would not be negligible in some cases. In this section, (a) the influence of the shape of the AFM probe on the measured cutting edge profiles, and (b) how to eliminate the influence of the shape of the AFM probe will be described. Furthermore, the evaluation method how to (c) measure the tip shape of the AFM probe will also be described.

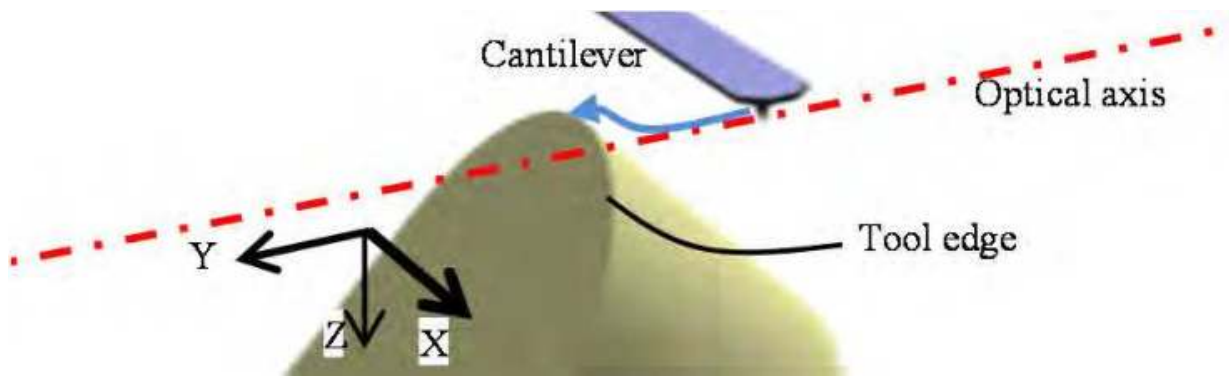

Fig. 2. Schematic of the cutting edge profile measurement by AFM

\subsection{Influence of the AFM tip geometry on profile measurement}

When we employ a contact-type sensor to carry out form accuracy measurements, the evaluated form data always contain a certain form error caused by the roundness or the tip shape of the styluses employed in the measurement setups. In case of the measurement of the cutting edge profile with an AFM, the evaluated cutting edge radius includes the roundness of the AFM tip. Figure 3 shows schematics of the paths of the AFM tip during the scanning over a surface with a step for the imaging. In the contact-mode measurement, AFM tip tries to trace the target surface. Basically, the AFM tip traces the target surface accurately when scanning over the relatively flat sections. On the other hand, the tip cannot trace the target surface accurately when scanning over a step-shaped surface because of its tip radius. When the shape of AFM tip is defined as $t(x)$, the reflected tip shape $p(x)$ can be described as

$$
p(x)=-t(-x)
$$

When the sample surface is defined as $f(x)$, the measured profile $g(x)$ can be described by a following equation (Villarrubla, 1997), while the Z-directional servo control of the AFM is properly performed.

$$
g(x)=\max _{x^{\prime}}\left[f\left(x^{\prime}\right)-t\left(x^{\prime}-x\right)\right]
$$

Namely, the series of the coordinate $(x, g(x))$ can be acquired by searching $x^{\prime}$, which gives the maximum value of $f\left(x^{\prime}\right)-t\left(x^{\prime}-x\right)$, at each $x$. As shown in Eq. (2), the acquired image 
profile $g(x)$ contains the information of the convex-shaped AFM tip $t(x)$. Such a phenomenon, in which the measured profile contains a certain influence of the measurement stylus, is called as "convolution" often used in the optical imaging. Furthermore, the operation reconstructing the original image from the measured image, which contains the information of the measurement stylus, is called "deconvolution". In case of the AFM measurement, the deconvolution would be possible if the shape of AFM tip $t(x)$ is known in advance, as shown in Fig. 3.

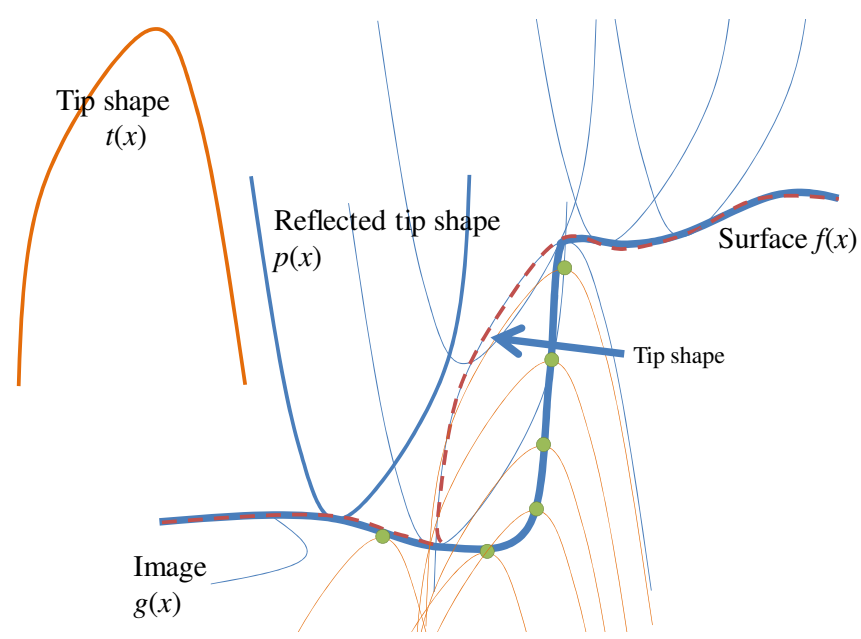

Fig. 3. Conceptual illustration of the imaging

Figure 4 shows a conceptual illustration of the reconstruction of the target surface from the measured results. Although the reconstructed surface sometimes does not represent the actual surface because there still remains some area where the AFM tip cannot contact, the reconstructed surface represents the target surface more accurately. The more precise the AFM tip shape is known, the more accurate the target surface profile can be measured. Although the discussion shown above was carried by using two-dimensional profiles in Figures 3 and 4 , the same discussion could be applied to the three-dimensional profiles obtained by AFM. It should be noted that the discussion above would be effective if there is no damage or wear on the AFM tip during the measurements.

There are some methods to observe or measure the roundness at the apex of the AFM tip. One of the most popular methods is to use scanning electron microscope (SEM). The SEM is the primary method to observe the sharp profile such as AFM tips (Bloo et al., 1999), and can be used to estimate the size of the tip apex. There are some other techniques to obtaining the shapes of tip apex. A tip characterizer, which has well-known shapes on its surface, is also used to estimate the AFM tip shape; namely, the shape of the tips will become known if there are well known structures to be imaged by the AFM. Moreover, the blind tip estimation, which is based on the theory of morphology, is another approach (Villarrubia, 1994; Dongmo et al., 2000). Because the convex of the image obtained by an AFM could have information about the tip, the tip shape can be estimated by correcting the AFM information. Furthermore, the implementation of the blind tip estimation has been explained in detail (Villarrubia, 1994). Some implementation is available on-line (Nečas \& 
Klapetek, n.d.). That is now modified to be applied to the tip with overhang (Tian et al., 2008).

In the following sections, we will discuss (A) the observation of the shape of AFM tip by SEM and (B) the estimation of the shape of AFM tip by tip characterizer.

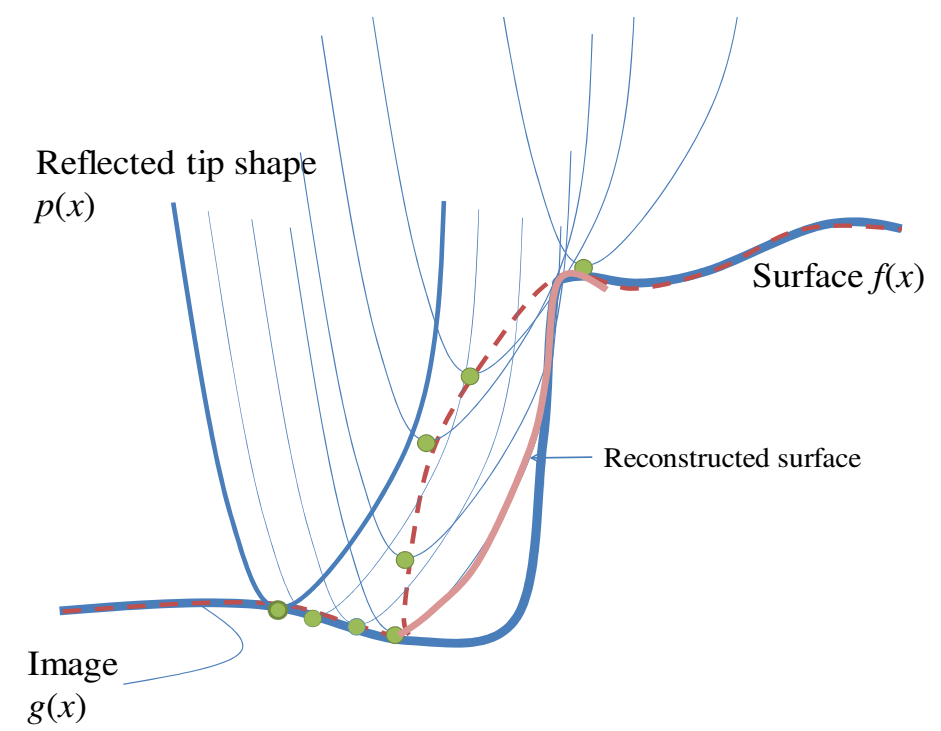

Fig. 4. Conceptual illustration of the reconstruction of the surface

\subsection{Observation of the shape of AFM tip by SEM}

Figures 5 show examples of the images of AFM tip and its apex measured by SEM. Figure 5(a) shows a whole shape of the AFM probe. The AFM probe has a conical-shaped sharp tip on the edge of the probe. Figure 5(b) is the magnified image of the same AFM tip shown in Figure 5(a). Although the shape of the AFM tip is not clear in the magnified images, the tip radius could be estimated around $30 \mathrm{~nm}$ from the SEM images. In fact, the AFM tip was said to be less than $20 \mathrm{~nm}$ in the specification sheet of the AFM probe, which roughly agrees with the measured tip radius.

It is not so difficult to acquire SEM image of the magnified AFM tip. Therefore, the SEM is one of the most popular methods among the various ones. However, it should be noted that the SEM observation itself could sometimes affect the shape of AFM tip. Figure 5(c) shows another SEM image of the same tip shown in Fig. 5(b). The SEM image in Fig. 5(c) was captured just a few minutes after the image in Fig. 5(b) was captured. The tip apex seemed to become dull from the original shape, which is considered to be due to a discharge or contamination on the AFM tip. It is well known that the SEM observation could easily damage or contaminate the very sharp tip without enough experiences (Egerton et al., 2004; Taniguchi et al., 1997). These results shown in Figs. 5 indicate that the radius of the AFM tip described here could be estimated slightly larger than which really was. However, it can be said that the SEM is one of common methods to evaluate the shape of AFM tip because of its ease. 


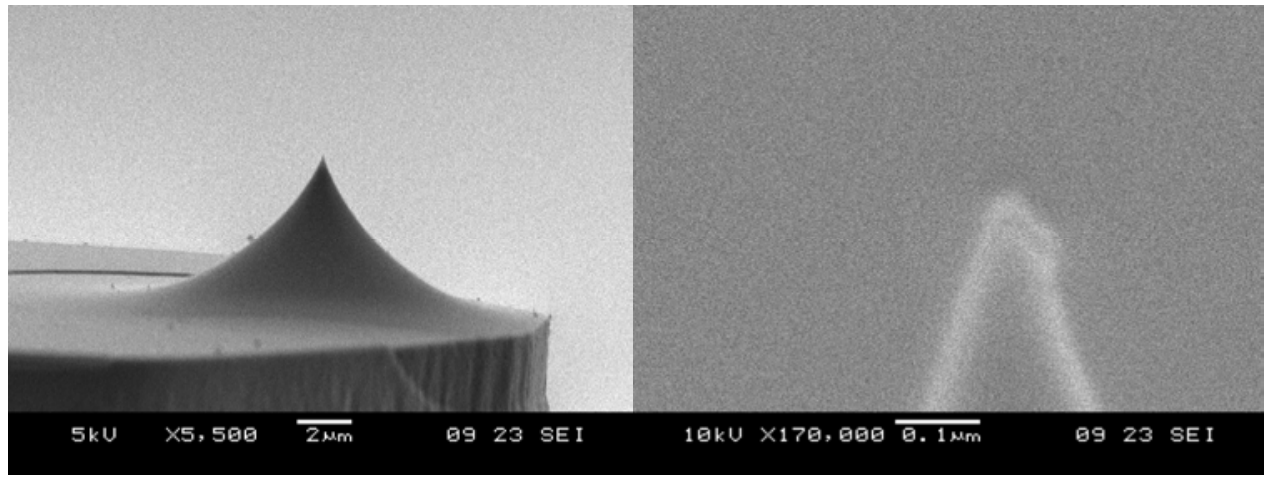

(a) Image of the tip

(b) Image of the tip apex magnified from (a)
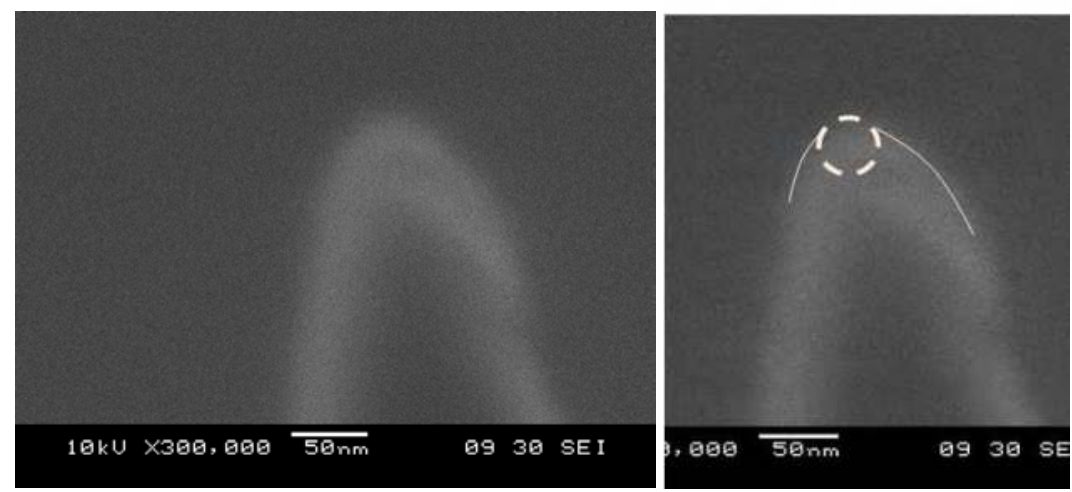

(c) Image of the tip apex re-measured a few minutes after the image (b) was captured

Fig. 5. SEM images of an AFM-tip, which were taken from the same AFM tip

\subsection{Estimation of the shape of AFM tip by tip characterizer}

Using a tip characterizer, which has well-known profile on its surface, is also used to estimate the shape of the AFM tip. A tip characterizer named TGG1, which has a very sharp edge, was used as an example. Figure 6 shows a schematic image of the profile on TGG1 surface. Several triangular steps were fabricated on a silicon wafer with a certain pitch, and the angles of each sharp edge were designed to be 70 degrees. Table 1 summarizes a detailed specification of TGG1. The radius of the sharp edge was fabricated to be less than $10 \mathrm{~nm}$, and the pattern pitch was also controlled to be $3.00 \mu \mathrm{m}$ with a tolerance of $\pm 0.05 \mu \mathrm{m}$.

Figure 7 shows a bird view of the profile of the tip characterizer measured by AFM. Figure 8 shows a cross-section image of the profile of the tip characterizer in Fig. 7, extracted at a certain X-axis position. To estimate the tip radius, a circle was fitted on the top of the measured profile in Fig. 8 by using least square method. The radius of the fitted circle shown in Fig. 8 was slightly less than $30 \mathrm{~nm}$. Actually, the value of the radius of the fitted circle could vary from 25 to $30 \mathrm{~nm}$ when the fitted region was selected arbitrary. Assuming that the edge radius of TGG1 is less than or exactly $10 \mathrm{~nm}$ referring to Table 1 , the tip radius of the AFM probe could be from 15 to $30 \mathrm{~nm}$, according to Eq. (1). On the other hand, the tip 
radius of the AFM probe should be less than $20 \mathrm{~nm}$ if we believe the datasheet of the AFM probe. However, considering the stability of the measurement instrument used here, it can be said that the AFM tip radius estimated by the tip characterizer roughly agrees with the value indicated in the datasheet.

It should be noted that the definition of edge radius may strongly affect to both the radius in datasheet and the radius measured by experiments; here we assume that there is an AFM tip, which tip width is $40 \mathrm{~nm}$, with dulled apex shape as shown in Fig. 9. Without any attention to the apex shape of the AFM tip, we might determine the tip radius is less than 20 $\mathrm{nm}$ even though the real tip radius of the AFM probe should be less than $20 \mathrm{~nm}$ in such a case; namely, the apex shape is locally flattened as shown in Fig. 9. Moreover, the region of the tip apex could affect to the determination of the tip radius. The definition of cutting edge radius will be discussed in the following section.

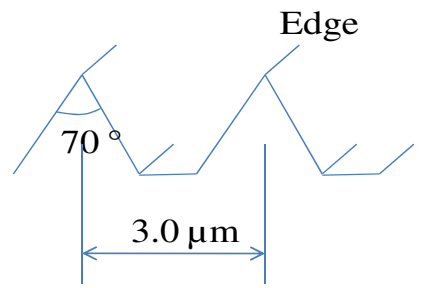

Fig. 6. Schematic of the structure on TGG1 surface

\begin{tabular}{|l|l|}
\hline Structure & Grating formed on Si wafer \\
\hline Pattern type & $\begin{array}{l}\text { One-dimensional array of triangular steps (in X- or Y-direction) having } \\
\text { precise linear and angular sizes }\end{array}$ \\
\hline Edge angle & 70 degrees \\
\hline Edge radius & $\leq 10 \mathrm{~nm}$ \\
\hline Period & $3.00 \pm 0.05 \mu \mathrm{m}$ \\
\hline
\end{tabular}

Table 1. Specification of the tip characterizer (TGG1)

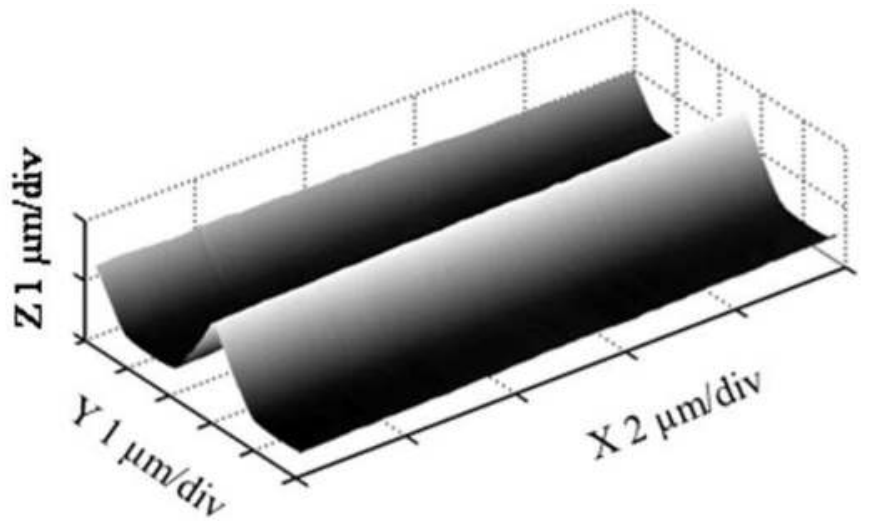

Fig. 7. Schematic of a tip characterizer "TGG1" 


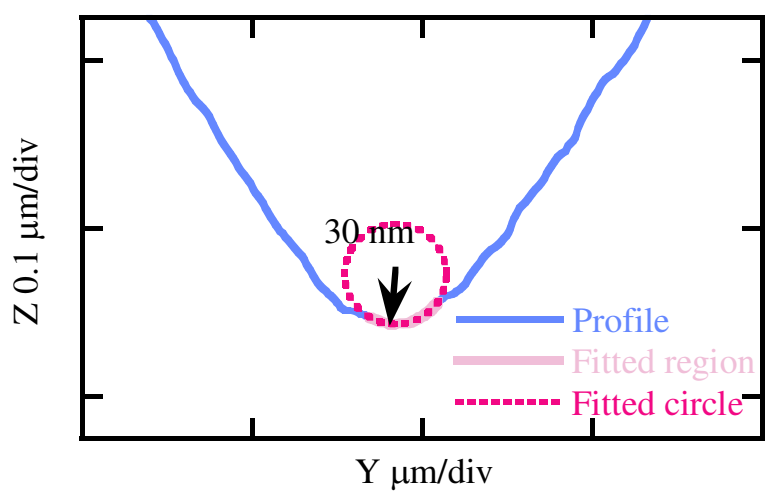

Fig. 8. Cross-section image of TGG1 in Fig. 7, at a certain X-axis position

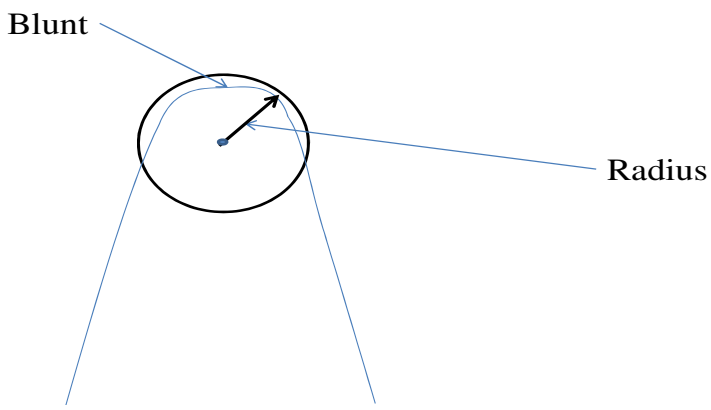

Fig. 9. Cross-sectional image of TGG1 in Fig. 5

\section{An evaluation method for cutting edge radius}

The value of the cutting edge radius is one of the most important parameters for the ultraprecision diamond cutting tool. There are some examples to obtain the cutting edge radius; some of them just fit a circle and employ its radius as the cutting edge radius, as shown in previous section (see Fig. 5(c)). In most cases, the definition of the cutting edge radius is not clear. Although methods shown in previous section will provide us pretty good estimations for the cutting edge radius, some concerns will still remain; where does the curvature starts?, which part of the curvature should we take as a radius?, and so on. Without a suitable method, we cannot define the cutting edge radius in a good repeatability. Therefore, many discussions on how to define the edge radius have been done, and some definitions have been proposed and utilized so far. In fact, however, we'll have to choose a proper method case by case, while taking both measurement accuracy and repeatability into consideration.

Based on such a background, in this section, the methods on how to handle the data of the cutting edge profile will be discussed. Because the cutting edge radius is named as "radius", a circle is usually fitted in many cases. Figure 10 shows a schematic of an ideal cutting edge profile. In Fig. 10, the cutting edge radius and edge angle were set to be $40 \mathrm{~nm}$ and 90 degrees, respectively. When the cutting edge radius shown in Fig. 10 is fitted by using the 
least squares method, the radius should be evaluated to be $40 \mathrm{~nm}$ because the applied profile was ideal and any noise component was not added onto the profile in Fig. 10. However, the situation becomes slightly different when the noise component is added, just like as a real case. Figure 11 shows a profile with a noise component. The noise component was added to the ideal profile shown in Fig. 10. The noise component is considered to affect the curve fitting for the cutting edge radius.

Therefore, a simulation was carried out to evaluate the influence of the noise component on the curve fitting. For the simulation, 100 lines of cutting edge profiles with noise component were numerically prepared in a personal computer. The noise components, which have the normal distribution with the standard deviation of $2 \mathrm{~nm}$, were generated by using random number generator. Then the circle fitting was carried out for numerically prepared each cutting edge profile.

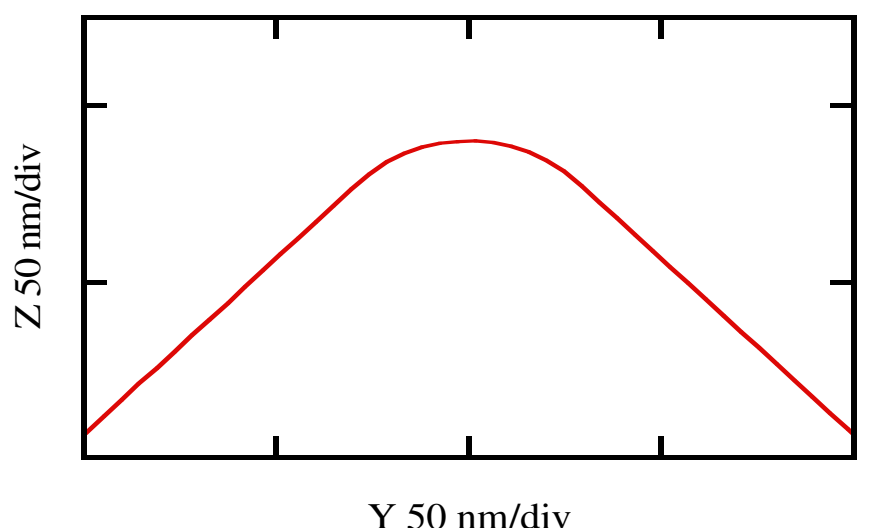

Fig. 10. An artificial edge profile having edge radius of $40 \mathrm{~nm}$

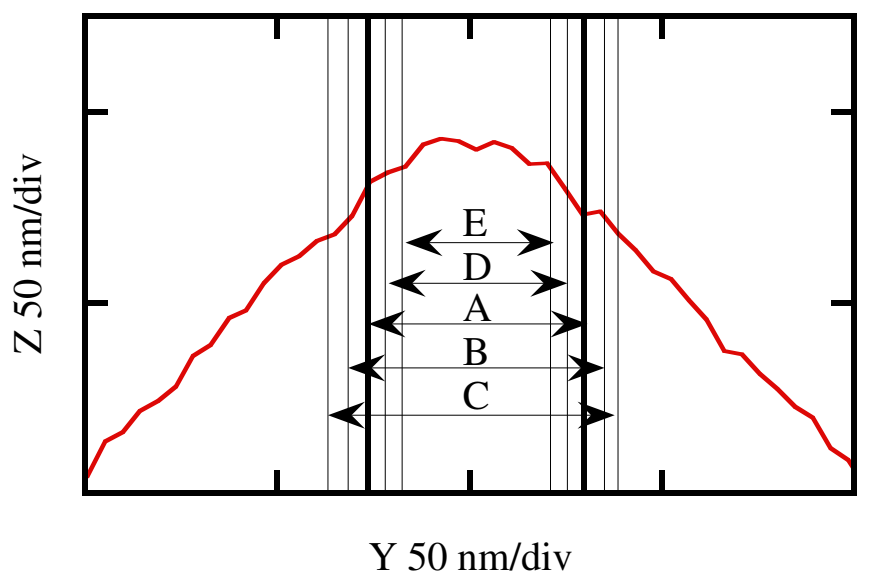

Fig. 11. An artificial edge profile having edge radius of $40 \mathrm{~nm}$ with the artificial noise with standard deviation of $2 \mathrm{~nm}$ 
When we carry out the circle fitting, a data region for the fitting will also affect the calculated cutting edge radius. Therefore, the data region was set to A, B, C, D and E as shown in Fig. 11, and the calculated cutting edge radius were compared.

Table 2 summarizes the average value and the standard deviation of the cutting edge radius calculated at each region from A to E for each 100 lines of cutting edge profiles. Focusing on the result at region $\mathrm{A}$, we can find out that the average of the calculated radius is about 30 $\mathrm{nm}$, with standard deviation of about $3 \mathrm{~nm}$. At each region, the average values of the calculated radius were smaller than $40 \mathrm{~nm}$, which is the radius of the ideal profile. As can be seen in Table 2, the wider data region resulted in larger cutting edge radius. On the other hand, results in Table 2 also tell us that the wider data region also resulted in the smaller standard deviation of the evaluated radius.

\begin{tabular}{|c|c|c|}
\hline Mark & Evaluated radius $(\mathrm{nm})$ & Standard deviation $( \pm \mathrm{nm})$ \\
\hline E & 15 & 4.2 \\
\hline D & 22 & 4.6 \\
\hline A & 30 & 3.4 \\
\hline B & 35 & 2.8 \\
\hline C & 38 & 2.1 \\
\hline
\end{tabular}

Table 2. Fitted circles size at each region (A, B, C, D and E are shown in Fig. 11)

In the case of the simulation shown above, the evaluated radius was considered to be estimated most properly when the region was selected as slightly larger than region C. However, no one can tell how wide we should set the data region in the actual measurement condition. If the evaluation range is set to be larger, a scale of the measurement not only data size but also measurement setup would become large. Anyway, the most important thing here is that the cutting edge radius could be evaluated to be very small or large, on purpose. Local disturbance of the profile due to noise components could cause the misevaluation. Therefore, the cutting edge radius should be evaluated without intentional operations as far as possible.

By the way, generally, the amount of the noise was defined as one sixth of the noise level of the instrument. If the value of standard deviation is determined as $\sigma$, about $99 \%$ of the whole sample is expected to be included in the range of $\pm 3 \sigma$ from the average. Of course, this assumption owes to the normal distribution such as Gaussian distribution. It should be noted that the resolution in Y-direction was about $4.6 \mathrm{~nm}$ due to quantization of the digital data, which would affect the results in Table 2.

There was an effort to define the cutting edge radius from the measured profile (Asai et al., 1999a; Asai et al., 1999b). A conic curve was fitted to the measurement result and the cutting edge radius was evaluated. There is another reason for selecting a conic curve instead of a circle; the set of conic curves includes ellipses, parabola and hyperbola. The circles are included by the set of ellipses. Therefore, conic curves are more general compared with circles. In case of the dulled edges, the profile is likely to be as shown in Fig. 12. In this case, an ellipse is considered to be the best choice to describe the tip part of this profile. The evaluation by using the circle might take small value compared with the local curvature radius of the edge top, depending on the region to be evaluated. Moreover, the status of the tool whether it is okay or already broken / worn off is important when we want to know the 
edge radius. However, it is not shown clearly if we use a circle for the fitting. Although there are other definitions proposed in some researches, of course, the edge is considered to be one of the regions marked as A and B in Fig. 12, depending on the case.

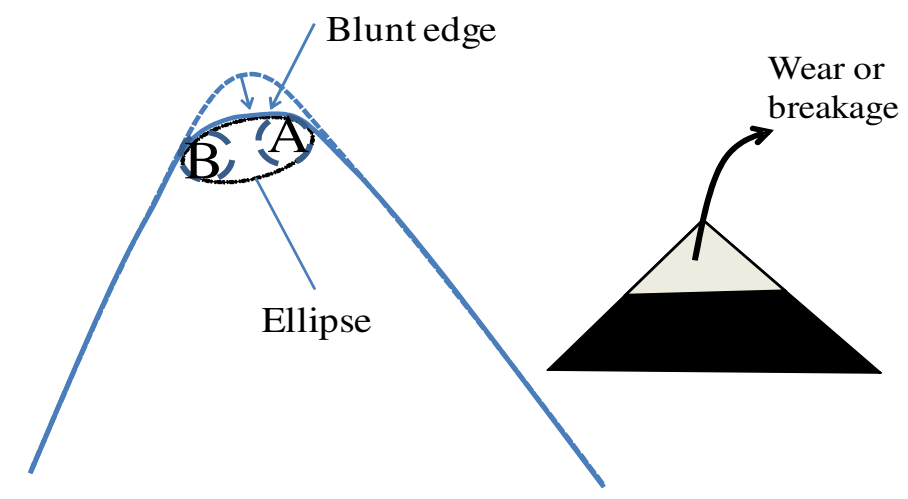

Fig. 12. An example of the blunt edge

According to the references, the actual operations can be described as follows (Asai et al., 1999a; Asai et al., 1999b). Figure 13 shows a schematic of a fitted curve function $F(x)$, which consists of two lines and one curve. Let $\left(x_{\mathrm{i}}, z_{\mathrm{i}}\right)$ be one of the coordinate of the measured data point. The evaluation function $\varepsilon$ can be described as follows:

$$
\varepsilon\left(p, Q_{S X}, Q_{T X}\right)=\frac{1}{n} \sum\left\{\left(F\left(x_{i}\right)-z_{i}\right)^{2}\right\}
$$

where $n$ is a number of the measurement points. If there are two crossing straight lines SO and OT as shown in Figure 13, the X- and Y-coordinates $(x, y)$ of the conic curves which are tangent to $\mathrm{SO}$ and $\mathrm{OT}$ at the points $\mathrm{S}$ and $\mathrm{T}$, respectively, can be expressed as follows.

$$
\begin{aligned}
& x(t, p)=\frac{X(t, p)}{W(t, p)} \\
& y(t, p)=\frac{Y(t, p)}{W(t, p)}
\end{aligned}
$$

while

$$
\begin{gathered}
X(t, p)=\left\{(1-p) Q_{S X}-2 p Q_{O X}+(1-p) Q_{T X}\right\} t^{2}-2\left\{(1-p) Q_{S X}-p Q_{O X}\right\} t+(1-p) Q_{S X} \\
Y(t, p)=\left\{(1-p) Q_{S Y}-2 p Q_{O Y}+(1-p) Q_{T Y}\right\} t^{2}-2\left\{(1-p) Q_{S Y}-p Q_{O Y}\right\} t+(1-p) Q_{S Y} \\
W(t, p)=2(1-2 p) t^{2}-2(1-2 p) t+(1-p)
\end{gathered}
$$

where $t$ and $p$ are the parameters. When $t=0$ and $t=1$, these equations represent the coordinate of the position $\mathrm{S}$ and $\mathrm{T}$, respectively. If the parameter $p$ is set to $0<p<0.5, p=0.5$ 
and $0.5<p<1$, the fitted conic curve by Eqs. (4)-(8) could be ellipse, parabola and hyperbola, respectively. If the parameter $p$ is getting smaller and smaller, $a / b$, the ratio of the major axis $a$ to the minor axis $b$ of the ellipse would be getting larger and larger.

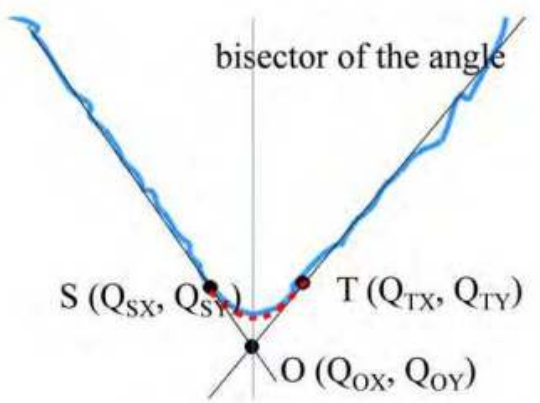

Fig. 13. Conceptual illustration of this first fitting

By using Fig. 13, we will explain how to carry out the fitting. Firstly, select the region to include an edge to be evaluated. Then fit the two lines by using the least squares method, while considering $\mathrm{Q}_{\mathrm{SX}}$ and $\mathrm{Q}_{\mathrm{TX}}$, which are the X-coordinates of the Point $\mathrm{S}$ and $\mathrm{T}$, respectively. Qsx and $Q_{T X}$ will be set in this order. It should be noted that not only parameter $p$ but also the coordinates of points $S$ and $T$ would be varied to search the best fitting.

Then find parameters $p, \mathrm{Q}_{\mathrm{Sx}}, \mathrm{Q}_{\mathrm{TX}}$ which minimize the parameter $\varepsilon$ in Eq. (3) so that the best set of the parameters $p, Q_{S x}$ and $Q_{T X}$, describing the actual profile, could be obtained. For the minimization, the following methods could be applied;

- Multidirectional search method (Sourceforge.net, n.d.)

- $\quad$ Nelder-Mead method (Sourceforge.net, n.d.)

- $\quad$ Genetic algorithm (Wall, n.d.)

After the fitting operations, draw a line which equally divides the angle between lines TO and $\mathrm{SO}$ as shown in Fig. 13. The curvature radius, at the point where the bisector line and the fitted curve, could be determined as one for the cross-sectional profile. However, in practice, the minimization algorithms can only find a local minimum and cannot always find a global minimum. That is a rule of the numerical minimization processes. Besides, a profile sometimes looks as if they do have a dulled edge by accidental noise peaks as shown in Fig. 14 (b).

Fig. 15 shows a histogram of the edge radii, which were calculated by using the previouslyprepared profile data in simulation. Because of these problems, the histogram of the evaluation looks like Fig. 15.

Comparing the result in Fig. 15 with the results in Table 2, it seems that the evaluation by fitting a circle looks better because of its small standard deviation. Actually, it is very interesting that the evaluation results with circle fitting do not have large deviation even if there is a profile as shown in Fig. 14(b). Having a large deviation with the fitting method shown above seemed to fits the lines more properly to a raw data. It does not mean that the evaluation of the edge radius is not possible at all, though. When we watch the histogram shown in Fig. 16 carefully, we can find out a peak around the value to be evaluated $(40 \mathrm{~nm}$ in this case). The value to be obtained is about the peak. 
There is a clear difference between a profile shown in Fig. 14(a) and (b). Therefore, a certain criterion is required to tell which evaluation is an outlier. Figure 16 shows the histogram of the edge evaluation as a function of parameter $p$ in Eqs. (4)-(8). The parameter $p$ is the main factor of the radius evaluation if the angle bisector is set properly. Therefore, in order to correct that there is a lower limit in the edge radius evaluation but not upper limit, the practical limit was applied to $p$. Assuming that the standard deviation of $p$ is $\sigma_{p}$ and the average value of $p$ is $\mu_{p}$, the lower limit of $p$ can be defined as

$$
p>\mu_{p}-\sigma_{p}
$$

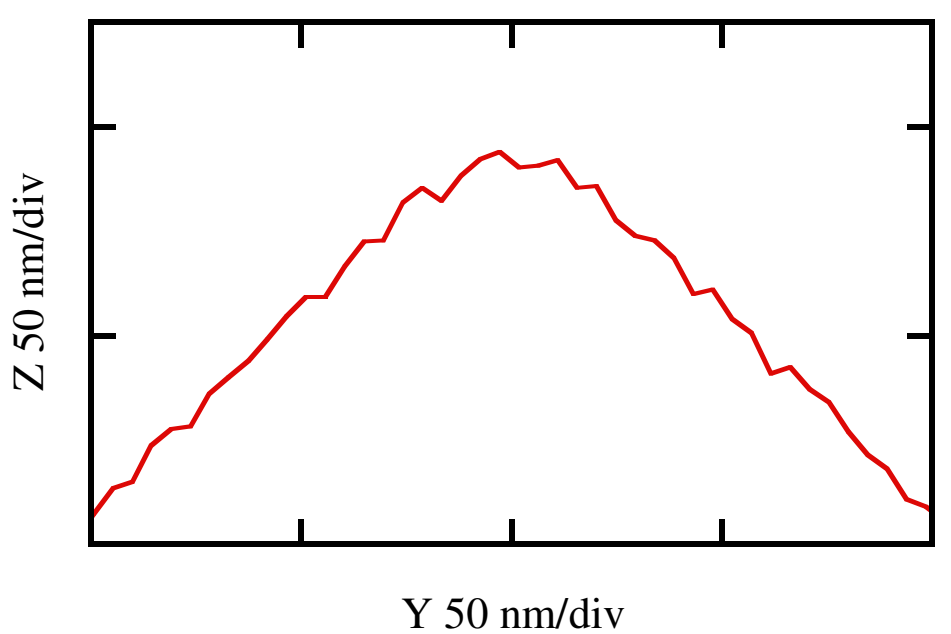

(a) A profile

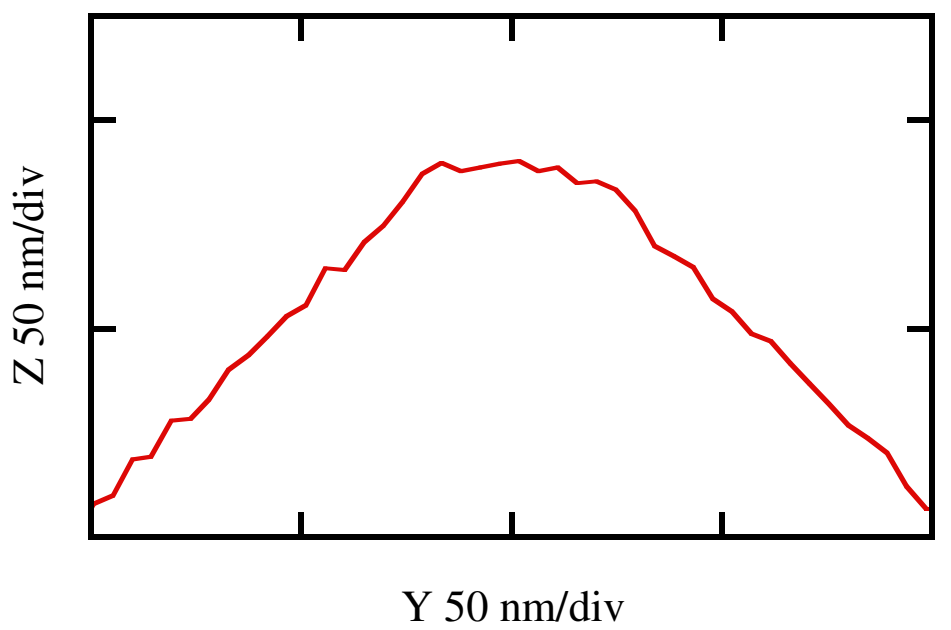

(b) A profile looked blunter than the profile in (a).

Fig. 14. Examples of the pseudo profiles. 
The evaluation that does not satisfy this limitation will be treated as an outlier. If the limitation in Eq. (9) is applied to the histogram shown in Fig. 16, the histogram of edge radius evaluation can be re-drawn as shown in Fig. 17. There still seemed to be two peaks around 0.040 and $0.065 \mu \mathrm{m}$, however, the average of the evaluations is $43 \mathrm{~nm}$, which is closer to the actual radius designed above. It should be noted that the limitation from upper side like as $p<\mu_{p}+\sigma_{p}$ could also be required if the histogram of $p$ has peak in upper side of the average, although the limitation from lower side is only mentioned above.

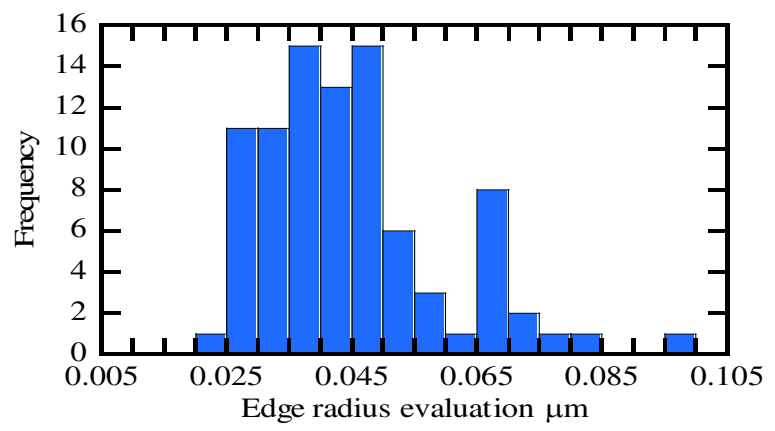

Fig. 15. Histogram of the edge radius evaluation

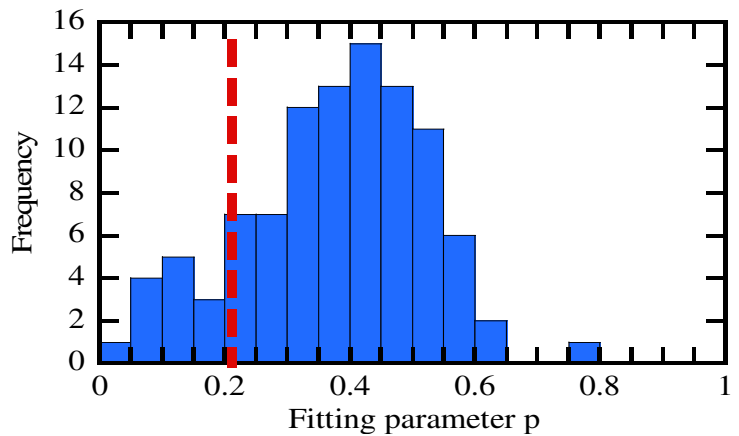

Fig. 16. Histogram of the edge radius evaluation as a function of fitting parameter $p$

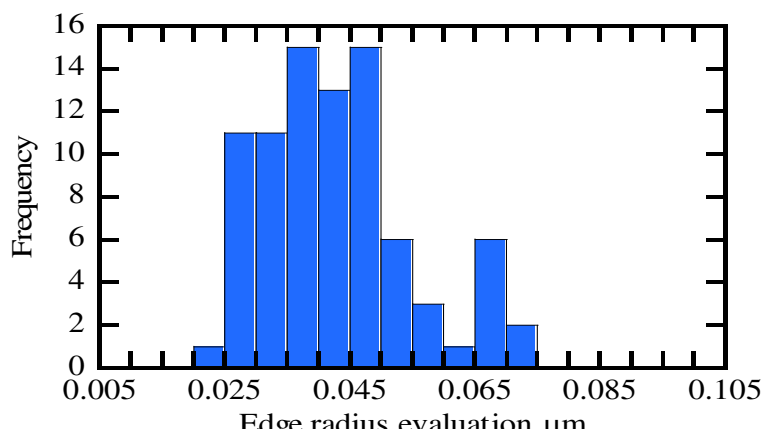

Fig. 17. Histogram of the edge radius evaluation when $p$ is limited 
Such tests have been carried out in some cases, while the Edge radius to be evaluated was changed from $10 \mathrm{~nm}$ to $60 \mathrm{~nm}$. If the radius to be evaluated is smaller than about $30 \mathrm{~nm}$, the parameter $p$ in Eqs. (3)-(7) has a possibility of taking a value about 0 . However, in this evaluation method, if the parameter $p$ takes 0 or 1 , it is not possible to evaluate the actual radius, because the evaluated radius becomes $\infty$ or 0 , in that case. Therefore, if the fitting parameter $p$ becomes 0 or 1 , the evaluations were removed from further calculations. The evaluations results were summarized as shown in Table 3 . The term "preprocess" in Table 3 means the removal of outliers. It should be noted that the the edge with edge radius of 10 $\mathrm{nm}$ could not be evaluated properly when the point interval is about $4.6 \mathrm{~nm}$, as mentioned in the previous section, because there should obviously be a lack of measure point.

If the parameter $p$ always takes small values, that mean the actual edge is really dulled like Fig. 14(a). Therefore, if $p$ is very small, there is a case that the edge should be considered separately; namely, only one of the edges A and B in Fig. 12 should be taken into consideration depending on the operator's decision, the region could be reselected to include one of two. It is thought that the former evaluation is more sensitive to the wear.

It should be noted that the $X$-direction represents vertical direction in the explanations shown above. In the case of the instrument mentioned in the previous section, the $X$ and $Y$ are exchanged. Therefore, the definition is switched to agree with that from here.

\begin{tabular}{|ccc|}
\hline Edge radiusnm & $\begin{array}{c}\text { Evaluation } \\
\text { (preprocessed) } \\
\mathrm{nm}\end{array}$ & $\begin{array}{c}\text { Standard deviation } \\
\text { (preprocessed) } \\
\text { nm }( \pm)\end{array}$ \\
\hline 10 & - & - \\
\hline 20 & 20 & 9 \\
\hline 30 & 29 & 10 \\
\hline 40 & 43 & 12 \\
\hline 50 & 48 & 11 \\
\hline 60 & 59 & 10 \\
\hline
\end{tabular}

Table 3. Evaluations results for each edge radius data (The edge radius to be evaluated is changed from $10 \mathrm{~nm}$ to $60 \mathrm{~nm}$ )

In practice, the region where the function $F(y)$ is fitted would be found in image to image, and line to line. Therefore, the region to be evaluated is selected like as follows, automatically.

At first, obtain the 2nd order of derivative along $y$ of the cross section image. In order to obtain the 2nd order of derivative along $y$, a "2nd order of least squares filter" is utilized (Savitzky \& Golay, 1964). The phrase "2nd order of least squares filter" means the repeating of the quadratic function fitting operations about the $y_{\mathrm{i}}$. Fig. 18 shows the simple explanation of the operation. In Fig. 18, a series of the marks " + " is the filtered data, which is more likely to preserve the height of the peaks than moving average. To carry out this filtering, a moving average type convolute function was selected.

Figure 19 shows the profile and the obtained 2nd order of derivative. The window was set to about $200 \mathrm{~nm}$. From the point where the 2 nd order of derivative takes maximum value to the point of the half of the maximum value are used as criterion. The lengths $\left(\mathrm{L}_{1}+\mathrm{L}_{1}{ }^{\prime}\right)$, and points $\mathrm{A}, \mathrm{E}, \mathrm{F}$ and $\mathrm{D}$ were defined as shown in Fig. 19. If there are two peaks in the 2nd 
order of the derivative, that means the edge or the AFM-tip is dulled. In such case, the window would be enlarged for the peak of the 2nd of derivative to take one obvious peak at first, aiming at the same effect as moving average to flat the sharp peaks.

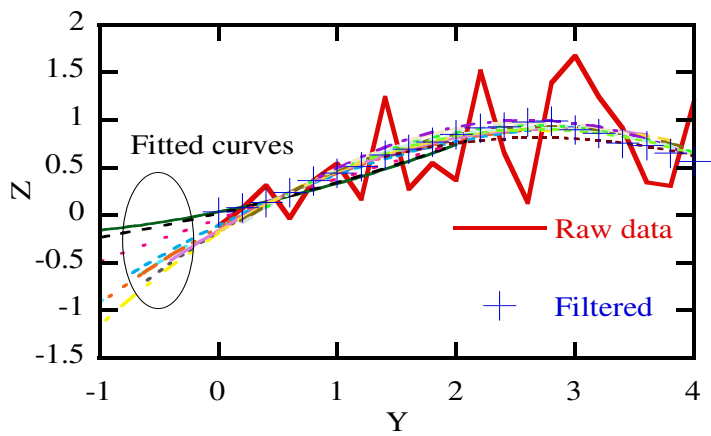

Fig. 18. Least squares filter used in finding the edge

Therefore, as a result, the lines $\mathrm{AB}$ and $\mathrm{CD}$ in Fig.19 would be fitted using least squares method to the data in the region between point $\mathrm{A}$ to point $\mathrm{E}$, and point $\mathrm{F}$ to point $\mathrm{D}$. A conic curve would be fitted in the region between point $\mathrm{A}$ to point $\mathrm{B}$ in the condition that the fitted conic curve tangent to the two lines, at first. As mentioned above, the point $\mathrm{E}$ and $\mathrm{F}$ would be replaced by the points $S$ and $\mathrm{T}$ in the fitting process, respectively. Moreover, the line $A B$ and line $C D$ would also be replaced depending on the points $S$ and $T$, respectively. The curvature radius of the cross point of the bisector of two straight lines $A B, C D$ and fitted conic curve would be called cutting edge radius, as previously mentioned.

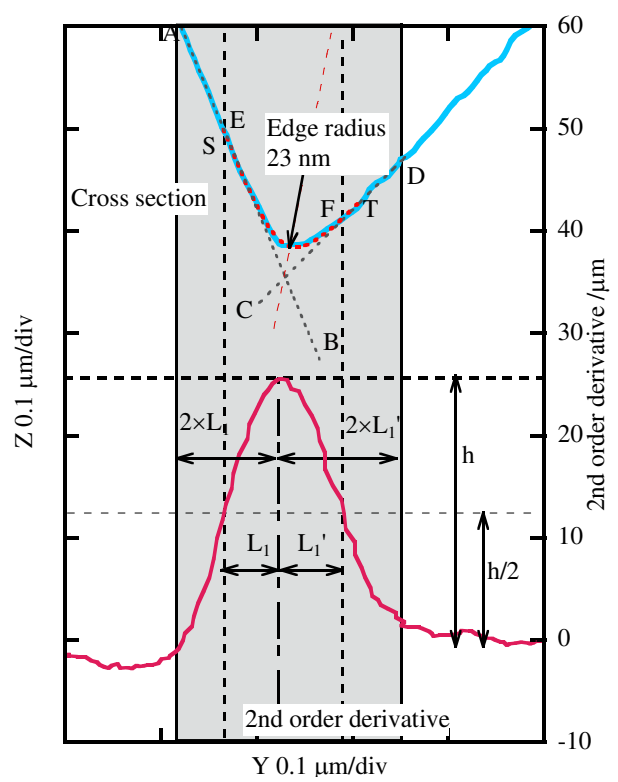

Fig. 19. Determination of the length used for the edge radius evaluation 


\section{Experiments}

\subsection{Measurement of a tool with nose radius of $0.2 \mathrm{~mm}$}

Some measurements of the diamond cutting tools were performed using a 3D cutting edge profile measuring instrument (Gao, 2010). The examples of the measurement results on the "real" profile of the cutting tools, in which the influences of the probe shape are eliminated, will be described. The measurements of the tools with nose radius of $0.2 \mathrm{~mm}$, and $1.5 \mu \mathrm{m}$ were performed. The results showed the clear edge between the rake face and clearance face, which could be used in the evaluation of the profile tolerance and the sharpness.

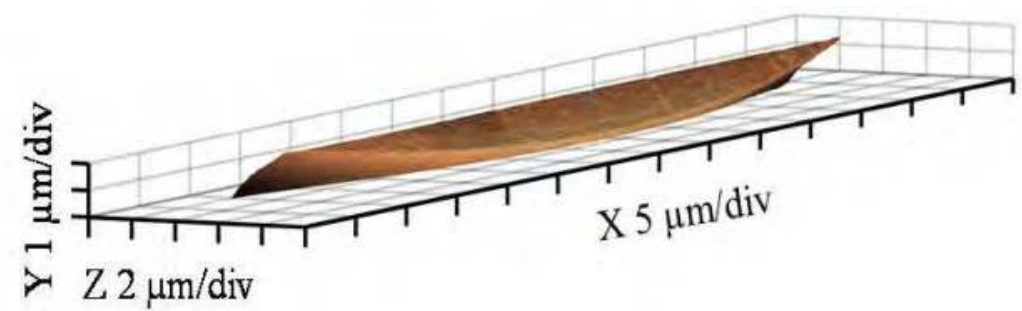

(a) Reconstructed image

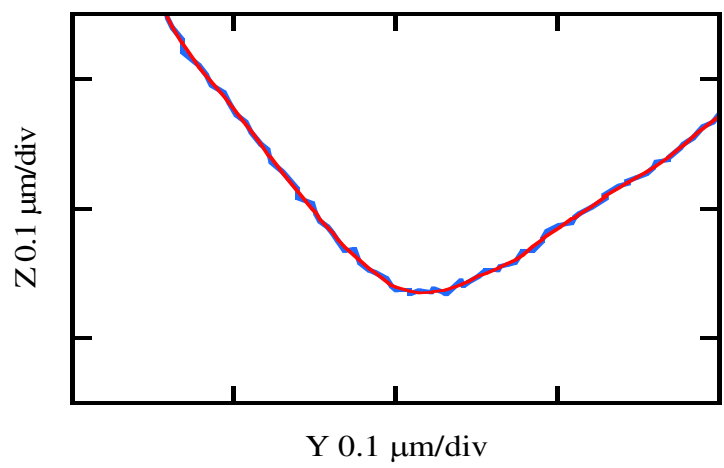

(b) A cross section

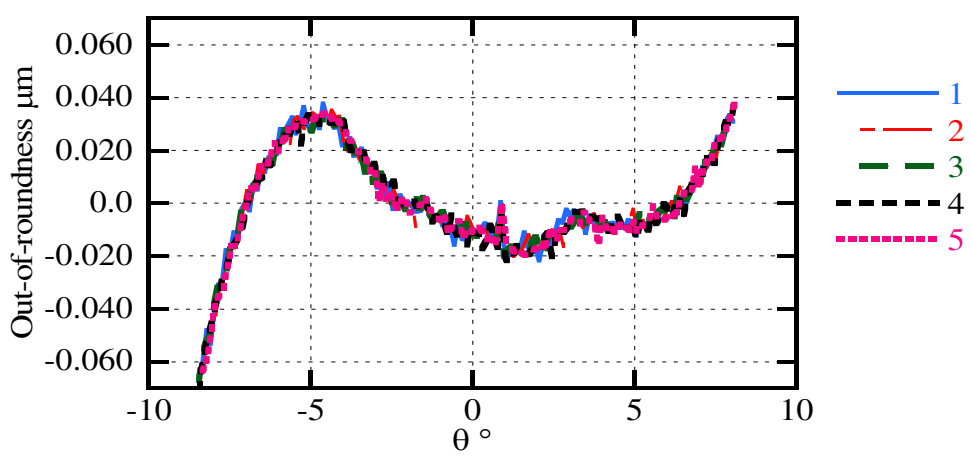

(c) Out-of-contour

Fig. 20. Measurement of a tool having nose radius of $0.2 \mathrm{~mm}$ 
Although the scan range of the PZT stages of AFM used in the measurement setup limits the measurable region, it is possible to measure the tool having nose radius of $0.2 \mathrm{~mm}$. Figs. 20 show examples of the measurement. Figure 20(a) shows a three-dimensional AFM image of the measured tool tip profile, and Fig. 20(b) shows its cross section image. The tool tip profile was measured five times, and both the averaged profile and non-averaged profile were imposed in Fig. 20(b). Figure 20(c) shows out-of-roundness obtained from Fig. 20(b). The cutting edge radius was evaluated to be $60 \mathrm{~nm}$ with standard deviation of $23 \mathrm{~nm}$ in the five-time measurement. The reason why the standard deviation became large is the large interval of the lines, which might make the evaluation of the local cutting edge radius impossible. The region to be evaluated is so small that the nose radius could not be evaluated with a repeatability on the order of nanometers (ranging from 0.1878 to 0.1880 $\mathrm{mm} / 5$ times), and the out-of-roundness seemed to be evaluated with repeatability up to 10 $\mathrm{nm}$. That is surprisingly good repeatability compared with the result shown above. The Zdirection positioning is a key to evaluate the tool having large nose radius because the movement of the AFM-tip on the sample surface in X-direction could not be easily translated to the Z-direction like cross talks in the case of the tools having large noses.

\subsection{Measurement of a tool with very small nose radius}

Measurement a tool with round nose radius of $1.5 \mu \mathrm{m}$ was performed. Figure 21 shows the evaluated cutting edge sharpness for the results of a measurement of a tool with nose radius of about $1.5 \mu \mathrm{m}$. The sectional profile of the cutting edge top in the $\mathrm{Y}-\mathrm{Z}$ plane was plotted. In Fig. 21, the results of repeated 3 times measurement were imposed. By using the method described in the previous section, the edge sharpness was evaluated to be approximately 39 $\mathrm{nm}$ with a standard deviation of $9 \mathrm{~nm}$. It should be noted that the influence of the tip radius of the AFM cantilever had not been removed from the result.

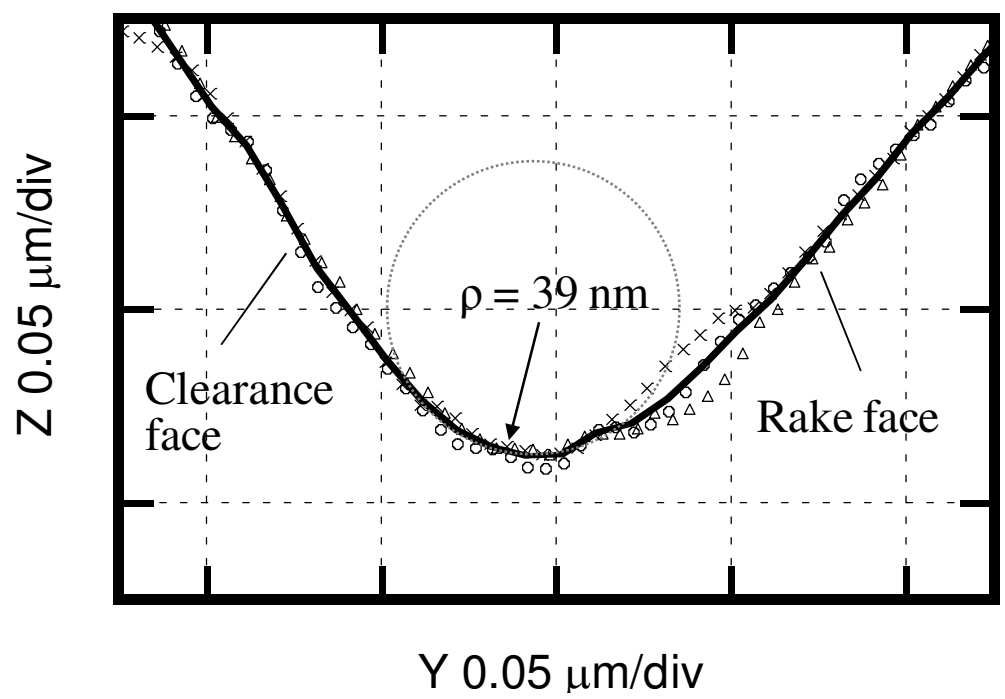

Fig. 21. Evaluated cutting edge sharpness for the results of a measurement of a tool with nose radius of about $1.5 \mu \mathrm{m}$. 


\section{Conclusions}

Measurements of the diamond cutting tools were performed using an AFM-based 3D cutting edge profile measuring instrument. The cutting edge profiles measured by AFM are affected by the shape of AFM probe. For nanofabrication, the influence of the shape of the AFM probe would not be negligible in some cases. That is why, in the second section, (a) the influence of the shape of the AFM probe on the measured cutting edge profiles, and (b) how to eliminate the influence of the shape of the AFM probe were described. Further, the evaluation method how to measure the shape of AFM probe and apply the acquired shape of the AFM probe to the measured cutting edge profile will also be described.

The value of the cutting edge radii is one of the most important parameters for the ultraprecision diamond cutting tool. There are some examples to obtain the cutting edge radii; some of them just put a circle and show the evaluated value. In most of the cases, the definition of the edge radius is not clear. Without a suitable method, we cannot define the edge radii of the cutting tools with a good repeatability. Throughout much discussion on how to define the edge radii, some definitions have been proposed and utilized. However, we'll have to choose a proper method case by case, while taking the measurement accuracy and the repeatability into consideration. In the third section, the methods how to handle the data of the cutting edge profile by the proposed method in the second section was discussed.

Moreover, the measurements of the tools with nose radius of $0.2 \mathrm{~mm}$ and $1.5 \mu \mathrm{m}$ were performed. The characteristic defects of the size of about $20 \mathrm{~nm}$ could be detected in each measurement. The results show the clear edge between the rake face and clearance face, which could be used in the evaluation of the profile tolerance or the sharpness. And in the cases of the tools with nose radii of micrometers, the repeatability of nose radius evaluation and the out-of-contour are good. That is about $\pm 10 \mathrm{~nm}$ level. Even in other cases, if only the out-of-roundness is concerned, not the radius of the nose, the order of the repeatability is on that level. Like them, by using the constructed instrument the evaluation of the cutting edge radii and the edge contour encompassing the nose radii and out-of-roundness are possible. This instrument was found to be useful for the evaluation of not only the diamond tool but also the tactile stylus tip.

\section{References}

Asai, S., Taniguchi, Y., Horio, K., Kasai, T., \& Kobayashi, A. (1990). Measuring and Analysys on Cutting Edge Radius of Single Point Diamond Tools using Newly Developed Scanning Electron Microscope (SEM), Journal of JSPE, Vol. 56, No. 7, pp. 145-150. (in Japanese)

Asai, S., Taniguchi, Y., Horio, K., Kasai, T., \& Kobayashi, A. (1990). Measuring the Very Small Cutting-Edge Radius for a Diamond Tool Using a New Kind of SEM Having Two Detectors, CIRP Annals, Vol. 39, No. 1, pp. 85-88.

Bloo, M. L., Haitjema, H., \& Pril, W.O. (1999). Deformation and wear of pyramidal, siliconnitride AFM tips scanning micrometre-size features in contact mode. Measurement, Vol. 25, pp. 203-2111.

Dongmo, L. S., Villarrubia, J. S., Jones, S. N., Renegar, T. B., Postek, M. T., \& Song, J. F. (2000). Experimental test of blind tip reconstruction for scanning probe microscopy, Ultramicroscopy, Vol. 85, pp. 141-153. 
Egerton, R. F., Li, P., \& Malac, M. (2004). Radiation damage in the TEM and SEM, Micron, Vol. 35, pp. 399-409.

Gao, W. (2010). Precision Nanometrology -Sensors and Measurement Systems for Nanomanufacturing-, Springer, ISBN 978-1-84996-253-7, London

Gao, W., Motoki, T., \& Kiyono, S. (2006). Nanometer edge profile measurement of diamond cutting tools by atomic force microscope with optical alignment sensor, Precision Engineering, Vol. 30, pp. 396-405.

Nečas, D., Klapetek, P. (July 2011). Gwyddion - Free SPM (AFM, SNOM/NSOM, STM, MFM, ...) data analysis software Users Guide, In: Gwyddion user guide, 05.07.2011, Available from http:/ / gwyddion.net/documentation/user-guide-en/

Savitzky, A., \& Golay, M. J. E. (1964). Smoothing and Differentiation of Data by Simplified Least Squares Procedures, Anal. Chem., Vol. 36, No. 8, pp. 1627-1639.

Sourceforge.net (July 2011). Documentation: GNU Octave and the Octave-Forge packages, In: Octave-Forge, 05.07.2011, Available from http://octave.sourceforge.net/ optim/function/fmins.html

Taniguchi, J., Miyamoto, I., Ohno, N., \& Honda, S. (1997). Utilizing of hydrocarbon contamination for prevention of the surface charge-up at electron-beam assisted chemical etching, Nuclear Instruments and Methods in Physics Research B, Vol. 121, pp. 507-509.

Tian, F., Qian, X., Villarrubia, J. S. (2008). Blind estimation of general tip shape in AFM imaging, Ultramicroscopy, Vol. 109, pp. 44-53.

Villarrubla, J. S. (1997). Algorithms for Scanned Probe Microscope Image Simulation, Surface Reconstruction, and Tip Estimation. Journal of Research of the National Institute of Standards and Technology, Vol.102, pp. 425-454.

Villarrubia, J. S. (1994). Morphological estimation of tip geometry for scanned probe microscopy. Surface Science, Vol. 321, pp. 287-300.

Wall M. (July 2011). GAlib: Matthew's Genetic Algorithms Library, In: GAlib, 05.07.2011, Available from http:/ / lancet.mit.edu/ga/ 


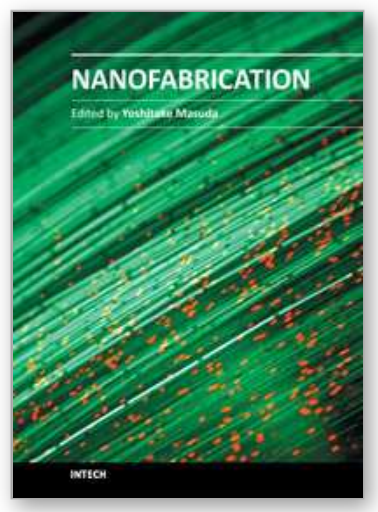

\author{
Nanofabrication \\ Edited by Dr. Yoshitake Masuda
}

ISBN 978-953-307-912-7

Hard cover, 354 pages

Publisher InTech

Published online 22, December, 2011

Published in print edition December, 2011

We face many challenges in the 21st century, such as sustainably meeting the world's growing demand for energy and consumer goods. I believe that new developments in science and technology will help solve many of these problems. Nanofabrication is one of the keys to the development of novel materials, devices and systems. Precise control of nanomaterials, nanostructures, nanodevices and their performances is essential for future innovations in technology. The book "Nanofabrication" provides the latest research developments in nanofabrication of organic and inorganic materials, biomaterials and hybrid materials. I hope that "Nanofabrication" will contribute to creating a brighter future for the next generation.

\title{
How to reference
}

In order to correctly reference this scholarly work, feel free to copy and paste the following:

Yuki Shimizu, Takemi Asai and Wei Gao (2011). Evaluation of Nanometer Cutting Tool Edge for Nanofabrication, Nanofabrication, Dr. Yoshitake Masuda (Ed.), ISBN: 978-953-307-912-7, InTech, Available from: http://www.intechopen.com/books/nanofabrication/evaluation-of-nanometer-cutting-tool-edge-fornanofabrication

\section{INTECH}

open science | open minds

\section{InTech Europe}

University Campus STeP Ri Slavka Krautzeka 83/A 51000 Rijeka, Croatia Phone: +385 (51) 770447

Fax: +385 (51) 686166 www.intechopen.com

\section{InTech China}

Unit 405, Office Block, Hotel Equatorial Shanghai No.65, Yan An Road (West), Shanghai, 200040, China 中国上海市延安西路65号上海国际贵都大饭店办公楼 405 单元 Phone: +86-21-62489820

Fax: +86-21-62489821 
(C) 2011 The Author(s). Licensee IntechOpen. This is an open access article distributed under the terms of the Creative Commons Attribution 3.0 License, which permits unrestricted use, distribution, and reproduction in any medium, provided the original work is properly cited. 